\title{
Equivalent Permeability of Step-Lap Joints of Transformer Cores: Computational and Experimental Considerations
}

\author{
Nabil Hihat $^{1,2}$, Ewa Napieralska-Juszczak ${ }^{1,2}$, Jean-Philippe Lecointe ${ }^{1,2}$, Jan K. Sykulski ${ }^{3,4}$, Fellow, IEEE, and \\ Krzysztof Komeza ${ }^{4}$ \\ ${ }^{1}$ Univ Lille Nord de France, F-59000 Lille, France \\ ${ }^{2}$ UA, LSEE, F-62400 Bethune, France \\ ${ }^{3}$ School of Electronics and Computer Science, University of Southampton, Southampton SO17 1BJ, U.K. \\ ${ }^{4}$ Institute of Mechatronics and Information Systems, Technical University of Lodz, 90-924 Lodz, Poland
}

\begin{abstract}
The paper develops an efficient computational method for establishing equivalent characteristics of magnetic joints of transformer cores, with special emphasis on step-lap design. By introducing an equivalent material, the method allows the real three-dimensional structure of the laminated thin sheets to be treated computationally as a two-dimensional problem and enables comparative analysis of designs. The characteristics of the equivalent material are established by minimizing the magnetic energy of the system. To verify the proposed approach, a series of experiments have been conducted. First, the anisotropic characteristics of the step-lap were established, and then space components of the flux density at specified positions measured. This enabled detailed analysis of the flux distribution in the step-lap region, in particular the way in which the flux travels between the laminations close to the air-gap steps. Encouraging correlation between the homogenized 2-D model and experiment has been observed.
\end{abstract}

Index Terms-Anisotropic laminations, equivalent magnetic characteristics, magnetic measurements, step-lap joints.

\section{INTRODUCTION}

$\mathbf{T}$ HIS study aims at developing practical methods of analysis of magnetic fields in the step-lap joints of power transformers. Magnetic cores of power transformers are almost always made of anisotropic laminations. Full 3-D numerical modeling of such structures is computationally very demanding, both in terms of long execution times and excessive memory requirements.

The actual joint consists of thin anisotropic laminations $(0.18-0.35 \mathrm{~mm})$, of varied directions of rolling, and of air gaps. The main idea of the approach presented in this paper is to replace the complex three-dimensional structure of the joint by an "equivalent" material [1]-[4] homogenized in two or three dimensions. In recent years, there have been several such attempts described in literature, most employing an analytical 1-D solution along the thickness of the sheet. Multidimensional orthogonal decomposition of the flux distribution has been used [5] both for a linear [6] and a nonlinear model [7]. The normal and tangential fluxes (referred to as "perpendicular" and "parallel" in the references) have been considered with appropriate modifications to the tensor of reluctances and conductances; a diagonal tensor has been used. A mathematical homogenization approach, based on the multiple scale expansion theory, for determining the nonlinear and hysteretic equivalent properties of magnetic composites has been presented in [8] and [9]. Several methods reported in literature exploit experimental methods appropriate for determining magnetically nonlinear characteristics of electromagnetic devices [10], [11]. Some

Manuscript received February 03, 2010; revised August 09, 2010; accepted October 14, 2010. Date of publication October 28, 2010; date of current version December 27, 2010. Corresponding author: E. Napieralska-Juszczak (e-mail: Ewa.napieralskajuszczak@univ-artois.fr).

Digital Object Identifier 10.1109/TMAG.2010.2089800 authors have implemented numerical inverse techniques in order to reconstruct the material characteristic [12], [13].

However, no method appears to have been developed to offer sufficient accuracy to deal with lamination stacks. This has motivated the authors of this paper to explore the possibility of representing the real 3-D structure by an equivalent 2-D material with the view of establishing equivalent (and effectively "artificial") characteristics, especially to make them suitable for use in combination with commercial field modeling software.

This paper describes the first stage of the investigation with the objective of replacing the 3-D region with an equivalent 2-D structure [14]. This first model neglects the effects of hysteresis and the influence of the normal flux (i.e., crossing the laminations) on the characteristics, although it is accepted that its presence, and that of the associated induced currents, may have an effect on the magnetic field distributions [15]-[17]. Thus, in the following derivations of the equivalent characteristics it has been assumed that the normal flux density $B_{z}$ is negligible compared to the components $B_{x}$ and $B_{y}$. Consequently, the effects of eddy currents have been neglected too [18]. Such an approximation will inevitably cause some inaccuracies, as although the component perpendicular to the sheets is typically only a few percent (5\%-8\%) of the tangential components, it travels through large areas. Comprehensive analysis of the influence of the normal and tangential fluxes on the magnetic field distribution in various laminated structures has been undertaken by the authors in their previous publication [19]. Moreover, the homogenization of the material and the introduction of equivalent anisotropic characteristics make it possible to use the method in combination with commercial software where modifications to the source code are normally not available. The proposed approach has been applied to step-lap joints which are particularly tricky to model because of the existence of internal air gaps that require a very fine mesh. Similarly, measurements involving such joints are also difficult due to the narrow steps and thus limited access to place the search coils. Generally, modeling of any laminated joints is considered a challenge [20]-[23]. A characteristic feature of the step-lap joint is a "comb" displacement 


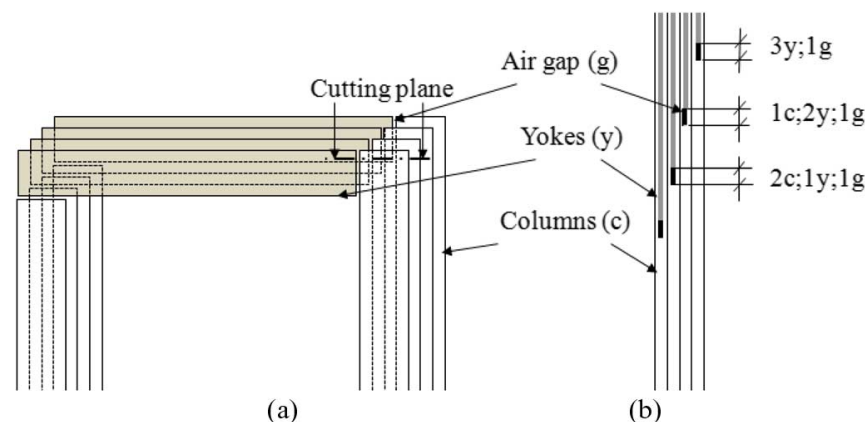

(a)

(b)

Fig. 1. (a) The step-lap joint of the transformer. (b) A cross section through the core corner.

("parallel" both vertically and horizontally) of the laminations in subsequent cycles of the core assembly [Fig. 1(a)]. In the region of an overlap, air gaps are created due to imperfections of the yoke and limb assembly (note that a term "column" is also used in literature, instead of a "limb," to describe the "vertical" section of the transformer core). Hence, in the core cross-section sequential presence of magnetic sheets (laminations) and gaps may be observed [Fig. 1(a)].

Regions containing in their cross-section laminations with different direction of rolling and at least one air gap will, from now on, be referred to as "virtual gaps." Fig. 1(b) depicts a core corner with four steps and different number of yoke and limb sheets. Using "c" for a column (limb), "y" for a yoke, and "g" for the gap, the notation $1 \mathrm{c} ; 1 \mathrm{~g} ; 2 \mathrm{y}$ implies the presence of one limb sheet, one gap, and two yoke sheets in the cross section. The "cutting plane" defining the "cross section" of Fig. 1(b) is marked on Fig. 1(a).

\section{EXPERIMENTAL SETUP}

A dedicated experimental rig was constructed to measure the anisotropic characteristics of a step-lap joint.

The magnetic core with a $90^{\circ}$ step-lap pattern is shown in Fig. 2. The magnetic circuit was built from rectangular sheets of anisotropic material from ThyssenKrupp Electrical Steel Company (M140-35S), of $800 \mathrm{~mm}$ length, $100 \mathrm{~mm}$ width, and 0.35 $\mathrm{mm}$ thickness. The power loss at $1.7 \mathrm{~T}$ at $50 \mathrm{~Hz}$ is $1.40 \mathrm{~W} / \mathrm{kg}$ [24]. We have assumed five cycles resulting in four "steps," with each step comprising two sheets. Five cycles is standard for unskewed corners and joints. Such designs lead to a smaller area taken by the "overlap" and allow the gaps to be spread over bigger space, resulting in better conditions for flux distribution. The neighboring layers of laminations are displaced by $5 \mathrm{~mm}$, which is typical for power transformers. The step-lap joints in all four corners have gaps of $1 \mathrm{~mm}$. In order to establish the equivalent characteristics of the joint, measurements needed to be taken for the laminations of different anisotropy angles. The anisotropy angle is measured between the direction of rolling and that of the flux density vector, with $0^{\circ}$ assumed when the two directions coincide. Measuring local flux density distributions for the individual steps necessitated insertion of search coils between the sheets.

The flux distribution in individual layers of the joint was measured. The values of the $x$ and $y$ components of the flux density were provided by specially wound search coils. To install such search coils, holes of $0.5 \mathrm{~mm}$ diameter were drilled at selected

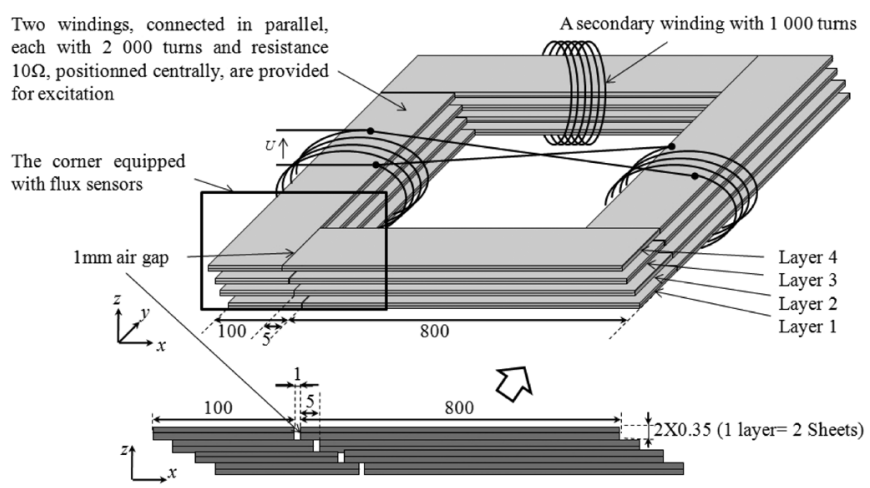

Fig. 2. The experimental magnetic core (all dimensions in $\mathrm{mm}$ ).

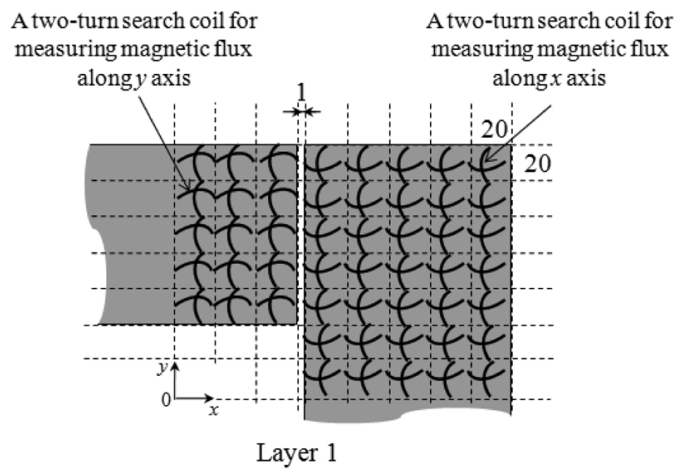

Fig. 3. Location of search coils in the first layer of the joint (all dimensions in $\mathrm{mm})$.

locations. The coils themselves have two turns of $0.1 \mathrm{~mm}$ diameter wire.

The position of the coils in the corner may be seen in Fig. 3 . This allowed measurements of the flux density to be taken for elements of $4 \mathrm{~cm}^{2}$ area at their geometric centers in the $x y$ plane. In order to reduce the gap between the layers, the holes in subsequent steps were shifted by $1 \mathrm{~mm}$. The effect of this shift is very small as the width of the coils is $20 \mathrm{~mm}$. The minimum gap needed to accommodate the coils is now double the wire diameter (of $0.1 \mathrm{~mm}$ ), rather than four times this diameter if no shift were present.

A simple measurement apparatus was used; before the values were recorded, signals from the coils were filtered and amplified by a factor of 1000 to obtain maximum amplitudes of $4 \mathrm{~V}$. A stabilizing circuit on the amplifier's input was used as a filter, consisting of a $50 \Omega$ resistor in parallel with a $1.2 \mu \mathrm{H}$ inductor. The magnetic field was excited by two coils (Fig. 2), each wound with 2000 turns, connected in parallel.

Finally, the effects of the drilled holes and installed coils (as shown in Fig. 3) were assessed. The actual "loss" of the surface area due to the presence of the holes was only about $0.3 \%$, despite the fact that there were many search coils (in fact 100 coils in the first layer and 132 in all remaining ones). In order to provide some quantitative measure of the possible errors, the global $B(H)$ curves were measured for the whole assembly before the drilling and then after installation of the coils. The comparison is shown in Fig. 4.

For flux densities below $0.8 \mathrm{~T}$, the two curves coincide. Some worsening of the magnetic properties due to the presence of the 


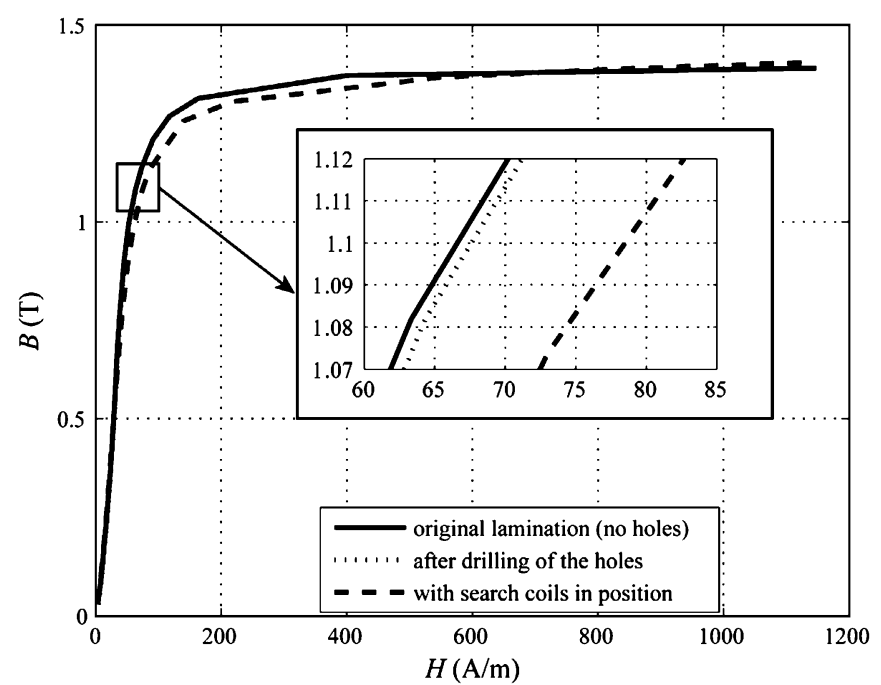

Fig. 4. Measured characteristics for the whole structure (before and after drilling of the holes and installation of the search coils).

holes and coils may be observed around the "knee point" of the $B(H)$ curve, but even in the worst affected region between 1.15 and $1.35 \mathrm{~T}$ the differences do not exceed $4.5 \%$. For higher flux densities, the three curves coincide again and there are no noticeable differences. The total flux density has been measured using a secondary winding of 1000 turns wound on one of the two limbs, the one not having the primary winding (Fig. 2).

\section{Equivalent Characteristics OF STEP-LAP JoINTS}

\section{A. Definition of the Corner Regions}

In the case of step-lap joints, the "steps" and "virtual gaps" are closely related as the gaps are "inside" the steps. The "iron filling factor" (how much of the cross section is taken by iron) of the part not interlaced decreases as we move closer to the edge of the core; this is due to the displacement of the yoke sheets of the same width. The edges of the joint have a comb shape, thus close to the edges structures (1c), (2c), (3c), (4c), (4y), (3y), $(2 y)$, and (1y) may be present. For each region of Fig. 5, the 3-D structure may be approximated by a homogenized material, for which an equivalent characteristic needs to be established. A description of the regions according to the numbering of Fig. 5, and following the notation from the Introduction, is summarized in Table I.

As an example, region 2 has two limb sheets (forming the edge of the magnetic circuit) and two gaps; region 11 is where the limb meets the yoke and in the cross section has two limb sheets, one yoke sheet and one gap, which in reality is the interior gap of the joint. For each of the fourteen regions of Table I and Fig. 5, the equivalent (homogenized) characteristics will be established to be used in combination with the 2-D field model of the magnetic circuit.

The homogenized equivalent structure consists of several laminations, with varied directions of rolling, and of air gaps. It is customary, when describing a family of $B(H)$ curves for different angles, to assume that $0^{\circ}$ is in the direction of rolling.

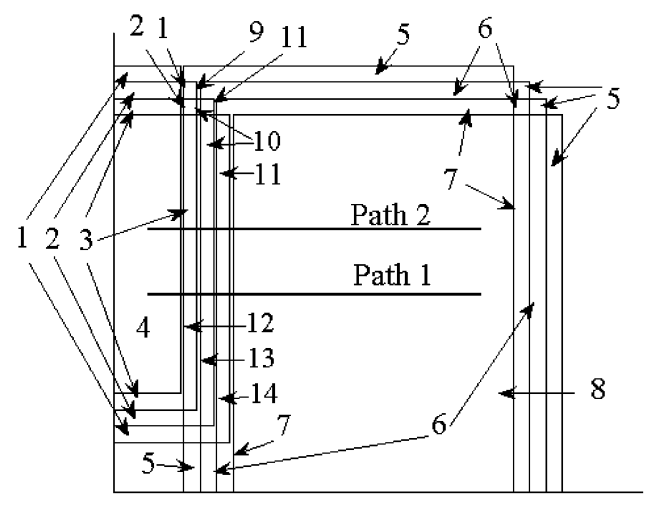

Fig. 5. A 2-D model of the core corner.

TABLE I

Description of the Regions Forming a Flat (2-D) Structure

\begin{tabular}{|c|c|c|c|}
\hline Region & Description & Region & Description \\
\hline 1 & $1 \mathrm{y} ; 3 \mathrm{~g}$ & 8 & $4 \mathrm{c}$ \\
\hline 2 & $2 \mathrm{y} ; 2 \mathrm{~g}$ & 9 & $1 \mathrm{c} ; 1 \mathrm{y} ; 2 \mathrm{~g}$ \\
\hline 3 & $3 \mathrm{y} ; 1 \mathrm{~g}$ & 10 & $1 \mathrm{c} ; 2 \mathrm{y} ; 1 \mathrm{~g}$ \\
\hline 4 & $4 \mathrm{y}$ & 11 & $2 \mathrm{c} ; 1 \mathrm{y} ; 1 \mathrm{~g}$ \\
\hline 5 & $1 \mathrm{c} ; 3 \mathrm{~g}$ & 12 & $3 \mathrm{c} ; 1 \mathrm{y}$ \\
\hline 6 & $2 \mathrm{c} ; 2 \mathrm{~g}$ & 13 & $2 \mathrm{c} ; 2 \mathrm{y}$ \\
\hline 7 & $3 \mathrm{c} ; 1 \mathrm{~g}$ & 14 & $1 \mathrm{c} ; 3 \mathrm{y}$ \\
\hline
\end{tabular}

This assumption is invalid in the case of an equivalent structure containing laminations of different directions of rolling; hence $0^{\circ}$ has been assumed to be coinciding with the yoke $(x$ coordinate in Fig. 2), while $90^{\circ}$ along the limb ( $y$ coordinate).

It has been assumed that same flux density exists in each yoke lamination of the region under consideration, in each limb sheet and in each air gap. There are $\mathrm{n}_{1}$ yoke sheets, $\mathrm{n}_{2}$ limb sheets and the number of laminations split by an air gap is $n_{0}$.

\section{B. Description of the Method}

The following argument exploits the system's natural tendency to achieve the minimum of the magnetic field energy. Two regions need to be considered: the virtual air gap and the overlapping area. The flux density of the equivalent structure of the air gap may then be defined as

$$
\left(n_{1}+n_{2}+n_{0}\right) \vec{B}=n_{1} \vec{B}_{1}+n_{2} \vec{B}_{2}+n_{0} \vec{B}_{0}
$$

where $\vec{B}$ is the equivalent flux density of the structure, $\vec{B}_{1}$ the density in the yoke sheet, $\vec{B}_{2}$ the density in the column sheet, and finally $\vec{B}_{0}$ the flux density in the air gap.

The energy stored in the virtual gap in the joint is

$$
W_{\mu}=\frac{1}{2} V_{s h}\left(n_{0} \nu_{0} B_{0}^{2}+n_{1} \nu_{1} B_{1}^{2}+n_{2} \nu_{2} B_{2}^{2}\right)
$$

where $\nu_{1}$ and $\nu_{2}$ are the reluctivities of the yoke and the limb, respectively, while $\nu_{o}=1 /\left(4 \pi 10^{-7}\right)$ and $V_{s h}$ is the lamination 
volume. In (2), the energy has been approximated using a linearized model which is a common approach in electromagnetic software packages. The objective function in the minimization process may be defined as

$$
f=n_{0} \nu_{0} B_{0}^{2}+n_{1} \nu_{1} B_{1}^{2}+n_{2} \nu_{2} B_{2}^{2}=\min
$$

subject to the constraint (1). The unknowns are the components of $\vec{B}_{1}$ and $\vec{B}_{2}$. The components $B_{0 x}$ and $B_{0 y}$ are found during the minimization process from

$$
\begin{aligned}
& B_{0 x}=\frac{n B_{x}-n_{1} B_{1 x}-n_{2} B_{2 x}}{n_{0}} \\
& B_{0 y}=\frac{n B_{y}-n_{1} B_{1 y}-n_{2} B_{2 y}}{n_{0}}
\end{aligned}
$$

where $B_{x}$ and $B_{y}$ are the components of the equivalent flux density.

For the overlapping area the flux density of the equivalent structure of the "step" may then be defined as

$$
\left(n_{1}+n_{2}\right) \vec{B}=n_{1} \vec{B}_{1}+n_{2} \vec{B}_{2} .
$$

The energy stored in any step of the overlap is given by

$$
W_{\mu}=\frac{1}{2} V_{s h}\left(n_{1} \nu_{1} B_{1}^{2}+n_{2} \nu_{2} B_{2}^{2}\right) .
$$

The objective function in the minimization process may be defined as

$$
f=n_{1} \nu_{1} B_{1}^{2}+n_{2} \nu_{2} B_{2}^{2}=\min .
$$

The unknowns are the components of $\vec{B}_{1}$. The components $B_{2 x}$ and $B_{2 y}$ of $\vec{B}_{2}$ are found during the minimization process from

$$
\begin{aligned}
& B_{2 x}=\frac{n B_{x}-n_{1} B_{1 x}}{n_{2}} \\
& B_{2 y}=\frac{n B_{y}-n_{1} B_{1 y}}{n_{2}} .
\end{aligned}
$$

It was initially imposed that vectors $\vec{B}_{1}$ and $\vec{B}_{2}$ are aligned with the rolling direction of the given layer at low values of the resultant flux density. At higher values of $\vec{B}$ uniform distributions are assumed. For the air gap, the starting values for $B_{1 x}$, $B_{1 y}, B_{2 x}$, and $B_{2 y}$ follow an initial assumption that magnetic flux goes entirely through the laminations avoiding the gaps. Moreover, for small values of flux density (smaller than $B_{p}$, where $B_{p}$ is the "knee point" in the direction of rolling) the flux goes mainly along the rolling direction, whereas for larger values (greater than $B_{p}$ ), $\vec{B}_{1} \approx \vec{B}_{2}$. A standard Hooke and Jeeves Direct Search Method [25], [26] has been used as it is known to be robust and insensitive to the starting (initial) point.

The equivalent reluctivity $\nu_{e q}$ of the homogenized replacement material representing the laminated system of the joint may be found by equaling the energies of the real and equivalent structures. Thus, for the air-gap region

$$
\begin{aligned}
\frac{1}{2}\left(n_{0}+n_{1}+n_{2}\right) & V_{s h} \nu_{e q} B_{e q}^{2} \\
= & \frac{1}{2} V_{s h}\left(n_{0} \nu_{0} B_{0}^{2}+n_{1} \nu_{1} B_{1}^{2}+n_{2} \nu_{2} B_{2}^{2}\right)
\end{aligned}
$$

and for the overlap region

$$
\frac{1}{2}\left(n_{1}+n_{2}\right) V_{s h} \nu_{e q} B_{e q}^{2}=\frac{1}{2} V_{s h}\left(n_{1} \nu_{1} B_{1}^{2}+n_{2} \nu_{2} B_{2}^{2}\right)
$$

where $\nu_{e q}$ is the equivalent reluctivity of the structure.

An important detail of the proposed algorithm is a suitable interpolation during calculation of the reluctivities $\nu_{1}$ and $\nu_{2}$; with this in mind, a method has been developed which allows these reluctivities to be established in terms of the modulus of the flux density and the angle of inclination of the vector with respect to the $x$ axis, while using the material characteristics for the angles $0^{\circ}, 90^{\circ}$, and $60^{\circ}$.

The equivalent characteristics for different regions have been computed by varying the equivalent flux density $B_{e q}$ from $0.01 \mathrm{~T}$ up to close to saturation (in fact up to the magnetic field strength of $2500 \mathrm{~A} / \mathrm{m}$ ); for each equivalent flux density the equivalent reluctivity was found through optimization. The equivalent characteristics were then computed for the angles $0^{\circ}$, $90^{\circ}, 30^{\circ}$, and $60^{\circ}$, on the basis of which values of $\nu$ were found for an arbitrary inclination angle of the flux density vector.

\section{COMPARISON OF THE NUMERICAL AND EXPERIMENTAL RESULTS}

\section{A. Numerical Results}

Equivalent static $B(H)$ curves have been computed-using, as the base, the characteristics measured on the Epstein apparatus [27] - for every region listed in Table I by applying the proposed homogenization method. The curves are drawn for various angles of inclination of the resultant vector B with respect to the horizontal line (coinciding with the yoke). A typical width of a "step" in the step-lap joint is around $3 \mathrm{~mm}$, the length of the air gap is between 0.15 and $0.5 \mathrm{~mm}$. Assuming careful assembly, the air-gap width may be taken as $0.25 \mathrm{~mm}$. In our experimental version, it was necessary to increase both the air-gap and the step width to allow search coils to be incorporated to measure the fields in all three directions in selected locations of the joint. Each "layer" consists of two sheets; between layers $0.2 \mathrm{~mm}$ gaps exist to allow for search coils. These gaps have been taken into account in both the computation of equivalent characteristics and in 3-D simulations. The describing parameter for the family of characteristics of the laminations, from which the joint is made, is the angle between the rolling and magnetization directions, whereas for the equivalent material it is the angle between the resultant flux density and the direction of the yoke ( $x$ in our case). The equivalent characteristics shown in Fig. 6 refer to the virtual gap region consisting of two yoke sheets, one limb sheet and one air gap, at four anisotropy angles of $0^{\circ}, 30^{\circ}, 60^{\circ}$, and $90^{\circ}$. Selected representative experimental and computed characteristics are summarized in Fig. 7. 


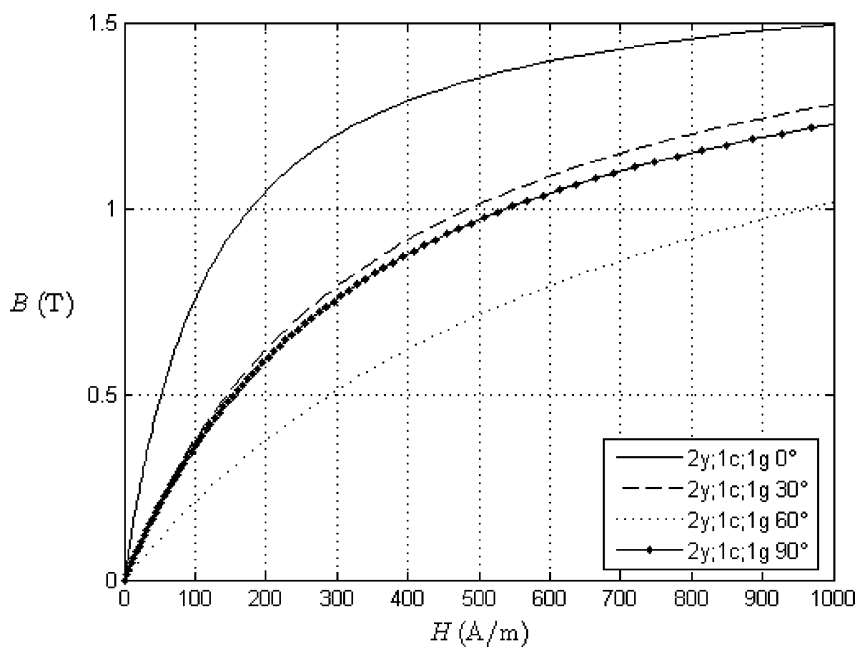

Fig. 6. Equivalent static $B(H)$ curves for the air-gap $(2 \mathrm{y} ; 1 \mathrm{c} ; 1 \mathrm{~g})$ at four different anisotropy angles.

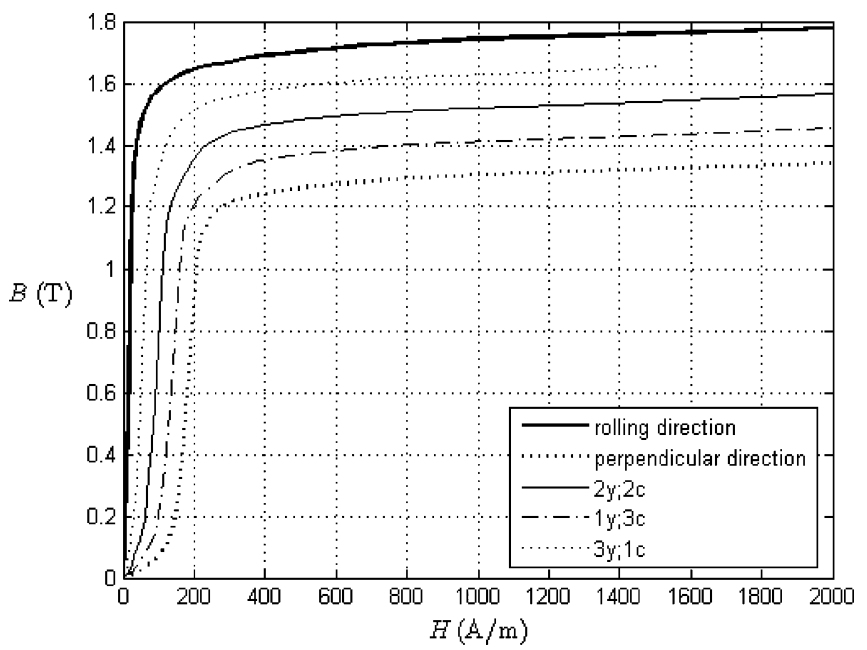

Fig. 7. Measured static characteristics for the lamination at angles of $0^{\circ}$ and $90^{\circ}$ and computed characteristics for various structures for the case of an angle of $0^{\circ}$.

The measured static curves refer to the laminations used in the assembly and are shown for both the rolling and perpendicular directions, while the computed values are given for the cases $1 \mathrm{y} ; 3 \mathrm{c}, 2 \mathrm{y} ; 2 \mathrm{c}$, and $3 \mathrm{y} ; 1 \mathrm{c}$ of the actual structure (regions 12, 13, and 14 in Table I). The computational model included the presence of insulation between the laminations. Fig. 7 should not be interpreted as a comparison between experiment and computation; instead the curves are merely superimposed to demonstrate that the characteristics of the different structures lie between the two extremes as given by the two directions of rolling. This result was to be expected as the actual structures are built from laminations of various combinations of anisotropy angles [2], [18]. The computed equivalent characteristics are then used to represent the 3-D effects using 2-D simulations.

The experimental rig has allowed for a comprehensive programme of measurements to be undertaken; the results presented here should not be treated as a review of various experiments and have been selected purely with the view of verifying the computational approach and demonstrating the merits of the proposed method.

The following observations can be made.

a) The equivalent material has less anisotropy than the original (real) laminated sheets. From Figs. 6 and 7, it transpires that the differences between the characteristics for the angles $0^{\circ}$ and $90^{\circ}$ are smaller for the equivalent material representing gaps and steps of the joint then those for the real structure. For example, at $\mathrm{H}=300 \mathrm{~A} / \mathrm{m}$, the difference between the flux densities in the directions $0^{\circ}$ and $90^{\circ}$ for the equivalent air gap $2 \mathrm{y} ; 1 \mathrm{c} ; 1 \mathrm{~g}$ is about $0.2 \mathrm{~T}$, while for the real sheet it is about $0.45 \mathrm{~T}$. However, these values are very local and have less impact on the overall accuracy.

b) The smaller the difference between the volumes of the relevant limb and yoke laminations, the less anisotropy the equivalent material exhibits; thus characteristics for different directions become closer to each other.

c) For higher values of flux density approaching saturation, for structures not containing air gaps, the characteristics tend to converge to similar values-this is not demonstrated in Fig. 6 as saturation has not been reached, but has been observed through other computations.

\section{B. 3-D Simulation}

In order to provide independent verification of the proposed approximations and gain more insight into the behavior of the magnetic field in the laminated structures, and in particular to study the effects of eddy currents, an attempt was made to model the systems studied using full 3-D finite-element code (a commercial package OPERA was used for this purpose). Such simulations are notoriously difficult for several reasons. The laminations are very thin, but they require several layers of elements to capture correctly the skin effect (a "rule of thumb" is to have at least three elements per penetration depth, which is about 0.26 $\mathrm{mm}$ in our case) - this leads to very small or very large aspect ratios (between 0.01 and 10) of the elements which may result in ill-conditioning of the solution matrix. Moreover, the insulation between laminations is extremely thin $(0.01 \mathrm{~mm})$ [24], which further adds to the problems. Such thin layers are normally ignored in simulations, but since we are focusing here on investigating accurately the laminated structure, such simplification could hardly be justified. Small and large elements may exist next to each other requiring careful grading of the mesh and inevitably leading to escalation of the total number of elements. In order to ensure fairness of comparisons, the 3-D model uses "real" geometry of the experimental setup, including the presence of the holes required for search coils.

However, some simplifications were still necessary; for example it was not possible to model the entire circuit of Fig. 3 (or rather a quarter of the circuit due to symmetry), instead the joint itself was placed in air, together with excitation coils, to force a certain amount of flux through the packet of laminations, with appropriate boundary conditions applied. Thus, it was assumed that normal flux density only existed at the two ends of the packet cross section, while the other surfaces were only allowed to have the tangential components (this is acceptable as with low to moderate values of flux density of the core 


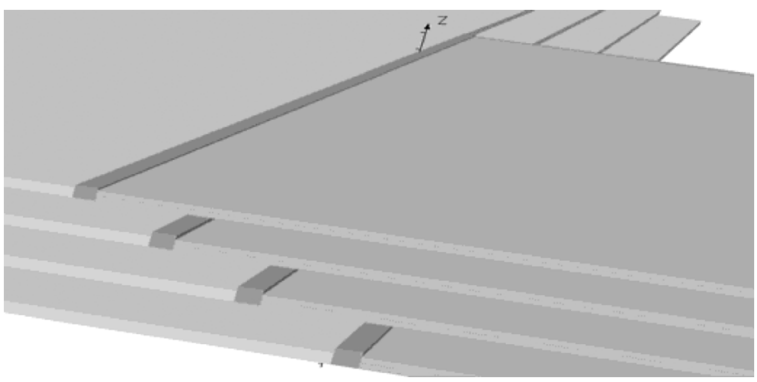

Fig. 8. 3-D model.

there will be little flux leaking out). The external boundary of the surrounding air space was positioned experimentally so that the change of the boundary condition on its edge would not affect the solution in the core. Each lamination (of $0.35 \mathrm{~mm}$ thickness) was modeled by four layers of elements with more, and smaller, elements near the $1 \mathrm{~mm}$ air gap between the limb and the yoke, as depicted in Fig. 8. The additional challenge, as mentioned before, was to incorporate the thin insulation between the sheets.

The resultant model consisted of 187406 nodes, 1278469 edges, and 1070924 linear tetrahedra. As expected, ill-conditioning was observed due to large variations in element volumes, extreme aspect ratios and not helped by the large difference between permeabilities of ferromagnetic core and air. This caused the Newton's method to fail. Thus, in order to achieve convergence, simple iterations had to be used (in fact, 93 iterations were needed) which resulted in very extended computing times, running into hundreds of hours.

The anisotropy was included using two magnetization curves, for the direction of rolling and in the perpendicular directions. Notwithstanding these difficulties, successful results have been obtained.

The main purpose of these 3-D simulations was to estimate the effect of induced currents, learn more about the field distributions, and provide independent verification of the proposed approximate approach in addition to the measurements.

Figs. 9-11 show distributions of $B_{x}$ and $B_{y}$ measured and computed using full 3-D numerical simulation, as well as the proposed approximate 2-D model using the homogenized equivalent characteristics. Before meaningful comparisons could be made, the results had to be processed as follows:

- flux densities obtained from both 3-D and 2-D calculations have been averaged over a patch $20 \mathrm{~mm} \times 20 \mathrm{~mm}$ equivalent to the area covered by a search coil to make computed results compatible with measurements; and

- flux densities resulting from a 3-D simulation were additionally averaged for each layer of laminations, that is along $z$ direction for given values of $x$ and $y$, to compare them with the 2-D results.

Figs. 9 and 10 show the distributions along the line $y=$ $90 \mathrm{~mm}$ and $x \in[10 \mathrm{~mm}, 151 \mathrm{~mm}]$, as depicted by path 1 in Fig. 5, while in Figs. 11 and 12 we have similar distributions along path 2 of Fig. 5 defined by $y=70 \mathrm{~mm}$ and $x \in$ $[10 \mathrm{~mm}, 151 \mathrm{~mm}]$. These positions have been selected as they refer to the centers of the overlapping between the limb and the

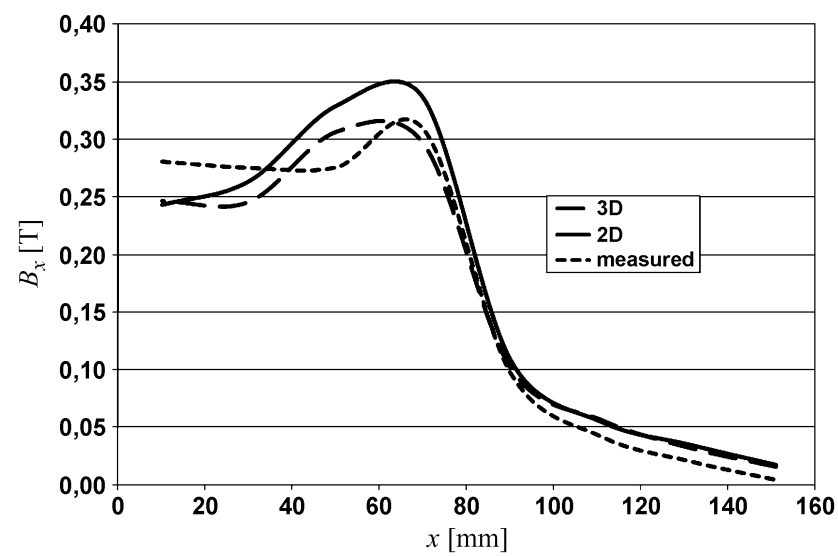

Fig. 9. Distribution of $B_{x}$ along path 1 (defined in Fig. 5): measured, computed using 3-D commercial software, computed using simplified 2-D homogenized model.

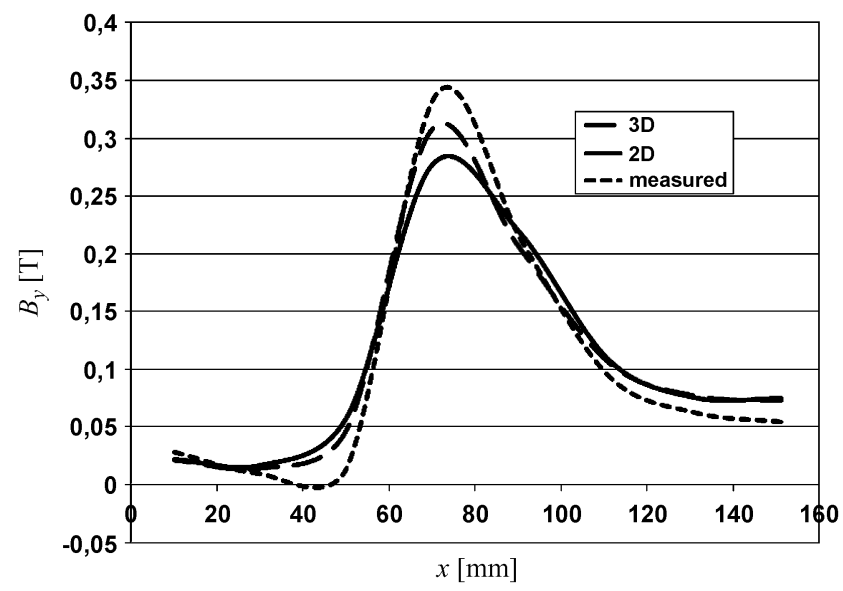

Fig. 10. Distribution of $B_{y}$ along path 1 (defined in Fig. 5): measured, computed using 3-D commercial software, computed using simplified 2-D homogenized model.

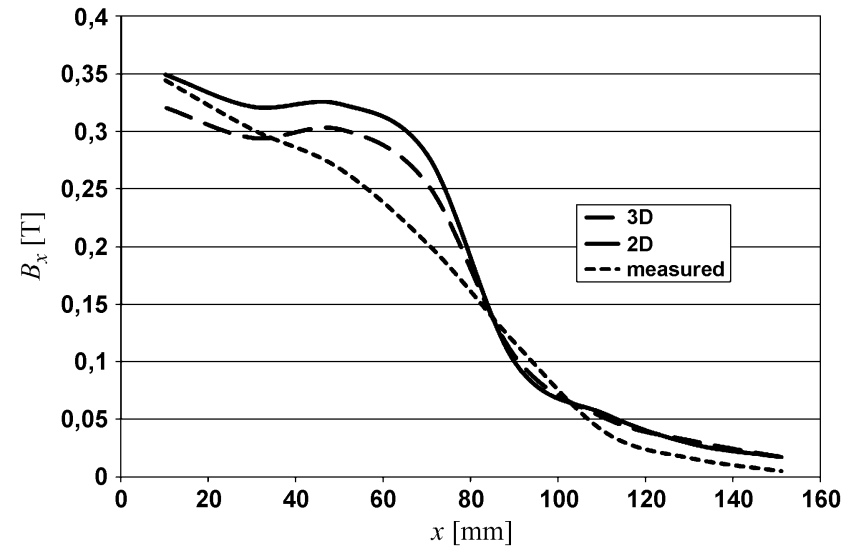

Fig. 11. Distribution of $B_{x}$ along path 2 (defined in Fig. 5): measured, computed using 3-D commercial software, computed using simplified 2-D homogenized model.

yoke. The point $(0,0,0)$ is in the top right-hand corner of the joint. The gap between the yoke and the column in the first layer occurs between $x=60 \mathrm{~mm}$ and $x=61 \mathrm{~mm}$, for the second layer between $x=65 \mathrm{~mm}$ and $x=66 \mathrm{~mm}$, and then between 


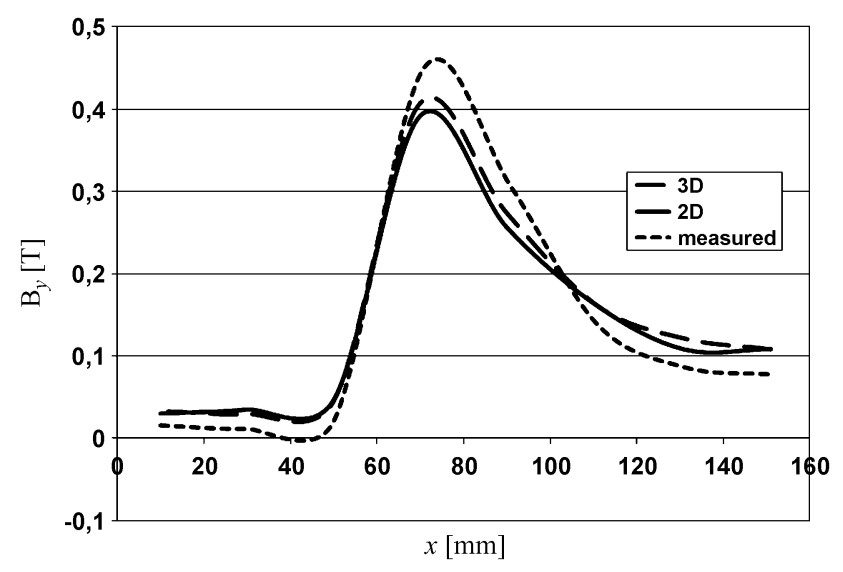

Fig. 12. Distribution of $B_{y}$ along path 2 (defined in Fig. 5): measured, computed using 3-D commercial software, computed using simplified 2-D homogenized model.

$x=70 \mathrm{~mm}$ and $x=71 \mathrm{~mm}$ and between $x=75 \mathrm{~mm}$ and $x=76 \mathrm{~mm}$ for the third and the fourth layer, respectively.

Overall, the comparison is encouraging. While the 3-D simulation is inevitably more accurate, the differences between the approximate "cheap" 2-D model using homogenized equivalent characteristics and measurements (or 3-D results) are small and similar to the differences between 3-D computation and experiment. Moreover, it has to be stressed that all three methods (including measurement) are subject to errors, thus it is impossible to conclusively state which values are absolutely correct. It can also be observed that the approximate 2-D method in some cases yields results which are closer to measurement than the 3-D simulation. On other occasions, the 3-D and 2-D results are very close indeed. A more detailed error analysis undertaken using the results presented in Figs. 9-12 has revealed that the 2-D homogenized model agrees with the 3-D simulations typically within 3\% to 5\% error, except close to areas where internal air gaps are present, where the error may increase to between $9 \%$ and $21 \%$ (worst case).

\section{CONCLUSION}

The proposed quasi-3-D method is fast and accurate if no internal air gaps are present. The measurements and 3-D simulations have demonstrated that in the region of the joints, as well as in the columns and yokes, the influence of the normal flux density (perpendicular to the laminations) is negligible, making the proposed homogenization technique accurate. However, in the air gaps between the column and the yoke, and the surrounding areas, neglecting the effects of eddy currents induced by the normal flux may be an oversimplification. Our current effort, and thus an extension of the work reported in this paper, is aimed at including the effects of the normal flux on the equivalent characteristics of the homogenized representative material. Overall, it can be argued that the proposed homogenization technique is fast and sufficiently accurate for design purposes. The experimental results verified the method to be a practical tool to predict field distributions in various types of overlapping joints and as an aid to design when anisotropic laminations are used.

\section{ACKNOWLEDGMENT}

The authors gratefully acknowledge the support from the program "Futurelec 8" under the initiative "Maîtrise Energétique des Entraînements Electriques" (MEDEE). This program, which includes ThyssenKrupp Electrical Steel, is sponsored by the region "Nord-Pas-de-Calais," the French Ministry (FRT), and the European funds (FEDER).

\section{REFERENCES}

[1] T. Nakata, K. Fujiwara, N. Takahashi, M. Nakano, and N. Okamoto, "An improved numerical analysis of flux distributions in anisotropic materials," IEEE Trans. Magn., vol. 30, no. 5, pp. 3395-3398, Sep. 1994.

[2] M. Pietruszka and E. Napieralska-Juszczak, "Lamination of T-joints in the transformer core," IEEE Trans. Magn., vol. 32, no. 3, pp. 1180-1183, May 1996.

[3] A. Bermúdez, D. Gómez, and P. Salgado, "Eddy-current losses in laminated cores and the computation of an equivalent conductivity," IEEE Trans. Magn., vol. 44, no. 12, pp. 4730-4738, Dec. 2008.

[4] J. Xu, A. Lakhsasi, Z. Yao, and V. Rajagopalan, "A practical modeling method for eddy-current losses computation in laminated magnetic cores," in Thirty-First IAS Annual Meeting, San Diego, CA, 1996, vol. IAS-96, no. 3, pp. 1532-1537.

[5] A. J. Bergqvist and S. G. Engdahl, "A homogenization procedure of field quantities in laminated electric steel," IEEE Trans. Magn., vol. 37, no. 5, pp. 3329-3331, Sep. 2001.

[6] J. Gyselinck and P. Dular, "A time-domain homogenization technique for laminated iron cores in 3D finite element models," IEEE Trans. Magn., vol. 40, no. 3, pp. 1424-1427, May 2004.

[7] J. Gyselinck, R. V. Sabariego, and P. Dular, "A nonlinear time-domain homogenization technique for laminated iron cores in three-dimensional finite-element models," IEEE Trans. Magn., vol. 42, no. 4, pp. 763-766, Apr. 2006.

[8] O. Bottauscio, M. Chiampi, and A. Manzin, "Homogenized magnetic properties of heterogeneous anisotropic structures including nonlinear media," IEEE Trans. Magn., vol. 45, no. 3, pp. 1276-1279, Mar. 2009.

[9] O. Bottauscio, V. Chiadopiat, M. Chiampi, M. Codegone, and A. Manzin, "Nonlinear homogenization technique for saturable soft magnetic composites," IEEE Trans. Magn., vol. 44, no. 11, pp. 2955-2958, Nov. 2008.

[10] G. Stumberger, T. Marcic, B. Stumberger, and D. Dolinar, "Experimental method for determining magnetically nonlinear characteristics of electric machines with magnetically nonlinear and anisotropic iron core, damping windings, and permanent magnets," IEEE Trans. Magn., vol. 44, no. 11, pp. 4341-4344, Nov. 2008.

[11] G. Stumberger, S. Seme, B. Stumberger, B. Polajzer, and D. Dolinar, "Determining magnetically nonlinear characteristics of transformers and iron core inductors by differential evolution," IEEE Trans. Magn., vol. 44, no. 11, pp. 1570-1573, Nov. 2008.

[12] D. Kowal, P. Sergeant, L. Dupre, and A. Van den Bossche, "Comparison of nonoriented and grain oriented mateial in an axial flux permanent magnet machine," IEEE Trans. Magn., vol. 46, no. 2, pp. 279-286, Feb. 2010.

[13] A. Abou Elyazied Abdallh, P. Sergeant, G. Crevecoeur, and L. Dupre, "An inverse approach for magnetic material characterization of an EI core electromagnetic inductor," IEEE Trans. Magn., vol. 46, no. 2, pp. 622-625, Feb. 2010

[14] G. Y. Xiao, A. J. Moses, and F. Anayif, "Normal flux distribution in a three-phase transformer core under sinusoidal and PWM excitation," IEEE Trans. Magn., vol. 43, no. 6, pp. 2660-2662, Jun. 2007.

[15] G. F. Mechler and R. S. Girgis, "Magnetic flux distributions in transformer core joints," IEEE Trans. Power Del., vol. 15, no. 1, pp. 198-203, Jan. 2000.

[16] H. Pfützner, C. Bengtsson, T. Booth, F. Löffler, and K. Gramm, "Three dimensional flux distributions in transformers cores as a function of package design," IEEE Trans. Magn., vol. 30, no. 5, pp. 2713-2727, Sep. 1994.

[17] M. A. Jones, A. J. Moses, and J. E. Thompson, "Flux distribution and power loss in the mitred overlap joint and power transformer cores," IEEE Trans. Magn., vol. MAG-6, no. 2, pp. 114-122, Mar. 1973. 
[18] N. Hihat, E. Napieralska-Juszczak, J. P. Lecointe, and J. K. Sykulski, "Computational and experimental verification of the equivalent permeability of the step-lap joints of transformer cores," in 7th Int. Conf. Computation in Electromagnetics (CEM 2008), Old Ship Hotel Brighton, U.K., Apr. 7-10, 2008

[19] N. Hihat, K. Komeza, E. Napieralska-Juszczak, J. P. Lecointe, and T. Niewierowicz, "Simplified models including eddy currents for laminated structures," Compel, vol. 5, no. 4, pp. 1033-1046, 2010.

[20] E. G. TeNyenhuis, R. S. Girgis, and G. F. Mechler, "Other factors contributing to the core loss performance of power and distribution transformers," IEEE Trans. Power Del., vol. 16, no. 4, pp. 648-653, Oct. 2001.

[21] G. F. TeNyenhuis and R. S. Girgis, "Measured variability of performance parameters of power \& distribution transformers," in Proc. IEEE PES Transmission and Distribution Conf. and Exposition, 2006, pp. 523-528.

[22] J. C. Olivares, S. V. Kulkarni, J. Cañedo, R. Escarela-Perez, J. Driesen, and P. Moreno, Impact of the Joints Design Parameters of the Wound Core in Distribution Transformer Losses. Calgary, Canada: ACTA Press, 2003, vol. 23, pp. 151-157, Issue 3.

[23] J. C. Olivares-Galván, P. S. Georgilakis, and R. Ocon-Valdez, "A review of transformer losses," Elect. Power Comp. Syst. J., vol. 37, no. 9, pp. 1046-1062, Sep. 2009.

[24] Thyssen Krupp Electrical Steel, "Our products-grain oriented electrical steel PowerCore," Product Catalogue Grain Oriented Electrical Steel PowerCore, ThyssenKrupp Electrical Steel GmbH, 2007.

[25] M. S. Bazaraa and C. M. Shetty, Nonlinear Programming Theory and Algorithms. New York: Wiley, 1979.

[26] R. Hooke and T. A. Jeeves, "Direct search," J. ACM, vol. 8, pp. 212-229, 1961.

[27] R. M. Bozorth, Ferromagnetism. New York: Van Nostrand, 1951.

Nabil Hihat received the M.Sc. degree in electrical engineering from Artois University, France, in 2007. He is currently a Ph.D. student at LSEE (Electrical Systems and Environment Research Laboratory), France.
Ewa Napieralska-Juszczak is Associate Professor at LSEE, University Artois France. Her research interests concentrate on modeling of magnetic fields in electrical devices, simulation of anisotropic materials, homogenization, and 3-D visualization. She is the author or joint author of over 150 papers.

Jean-Philippe Lecointe (M'04) was born in Béthune, France, in 1976. He received the M.Sc. degree from The Artois University, France. He received the Ph.D. degree in 2003.

He is Associate Professor at the Artois University and he joined the institute LSEE (Electrical Systems and Environment Research Laboratory), France. His research interests focus on electromagnetic design, efficiency, noise, and vibrations of electrical machines.

Jan K. Sykulski (M'93-SM'94-F'10) is Professor of Applied Electromagnetics at the University of Southampton, U.K. His research covers computational electromagnetics, applications of high temperature superconductivity, simulation of coupled field systems and design optimisation of electromechanical devices. He has published over 300 scientific papers. He is General Secretary of International Compumag Society, Editor-in-chief of COMPEL (Emerald) and member of Steering Committees of several international conferences.

Prof. Sykulski is Fellow of the IET, IoP, and BCS.

Krzysztof Komeza is Associate Professor at Technical University of Lodz, Poland. He specializes in electrical engineering, especially in theory of electromagnetic fields, efficient finite-element computations and coupled field computations, and electrical machines. He has done a number of consultancy works for electrical machines industry. He is author or joint author of over 160 publications. 